\title{
The one-and-a-half syndrome. Clinical correlation with a pontine lesion demonstrated by nuclear magnetic resonance imaging in a case of multiple sclerosis
}

\author{
C N MARTYN AND D KEAN \\ From the University Departments of Medicine and Radiology, Royal Infirmary, Edinburgh
}

SUMMARY Nuclear magnetic resonance (NMR) imaging has been used to visualise a localised area of demyelination in the dorsal pons of a patient who presented with an unusual disorder of eye movements, namely, the one-and-a-half syndrome. Judged clinically the patient made a nearly complete recovery, but little change was apparent in the NMR image six months later.

The unusual and remarkable disorder of eye movement consisting of a lateral gaze palsy in one direction and an internuclear ophthalmoplegia in the other was probably first described by Freeman et al.' and later dubbed the one-and-a-half syndrome by Fisher. ${ }^{2}$ In the fully developed syndrome one eye is in the midline and completely fails to move horizontally while the other can only abduct. Fisher attributed the syndrome to a single unilateral lesion affecting the centre for conjugate lateral gaze in the paramedian pontine reticular formation and the adjacent ipsilateral medial longitudinal bundle. This anatomical localisation has been confirmed at necropsy in several cases. Recorded causes of the syndrome include tumour, trauma, infarction, haemorrhage, and demyelination. ${ }^{34}$ We report a further case, occurring in a patient with multiple sclerosis, in which the lesion was successfully visualised during life by magnetic resonance imaging.

\section{Case report}

A 30-year-old woman presented with a two-week history of diplopia, unsteadiness of gait, and altered taste sensation. She had noticed, too, that her face had become asymmetrical. Examination revealed a complete horizontal gaze palsy to the left for voluntary and tracking eye movements and also for reflex

Correspondence to Dr C N Martyn, MRC Environmental Epidemiology Unit, University of Southampton, Southampton General Hospital, Southampton SO9 4XY. eye movements in response to head turning. A contralateral internuclear ophthalmoplegia was present: the left eye failed to adduct past the midline, and horizontal jerking nystagmus was apparent in the abducting right eye. Up-gaze was incomplete with gaze-evoked upbeat nystagmus, and convergence was impaired. Figs. 1-3 illustrate the horizontal gaze palsies. There was an incomplete left-sided facial palsy of lower motor neurone type, and taste sensation was lost on the left side of the tongue. Abdominal reflexes were absent, and both plantar responses were equivocally extensor.

Eight years earlier she had been investigated extensively but inconclusively for an episode of bilateral foot drop associated with paraesthesiae

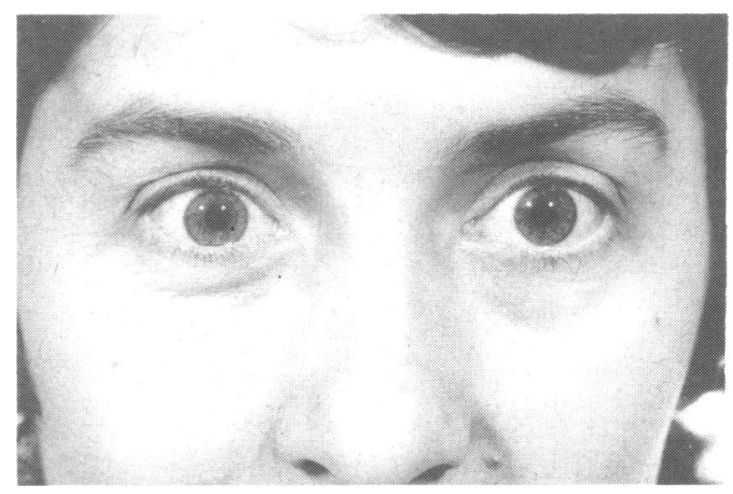

Fig. 1 Patient is attempting to look straight ahead. No abnormality of conjugate gaze is apparent. 


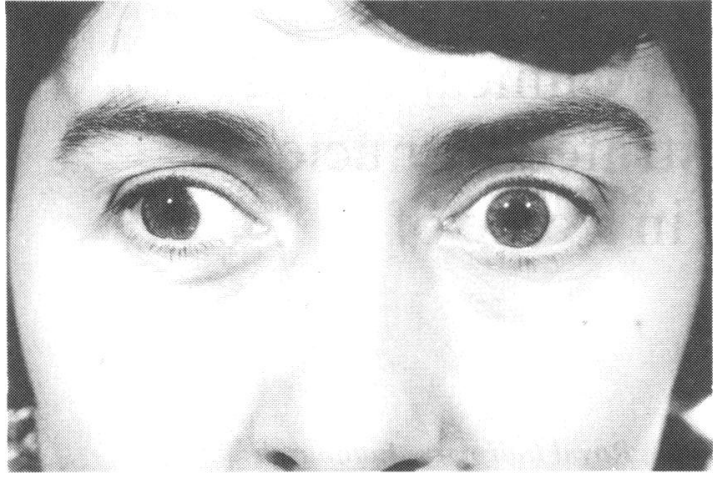

Fig. 2 Patient attempts to look right. The right eye abducts, but the left remains fixed at the midline.

affecting both lower limbs. Myelography had been normal and no abnormalities in the cerebrospinal fluid (CSF) had been detected. She had recovered fully within three months. Four years later she had experienced paraesthesiae and weakness affecting the whole of her left arm in association with difficulty in manipulating small objects with her left hand. No investigations had been undertaken, but she again made a full recovery within a few months. While this report was being prepared she sustained a typical attack of left-sided retrobulbar neuritis.

Investigations during her most recent illness were as follows: erythrocyte sedimentation rate $11 \mathrm{~mm} / \mathrm{h}$, antinuclear factor test negative, DNA binding $<5$ units/ml (normal less than 25 units/ml), Mantoux test

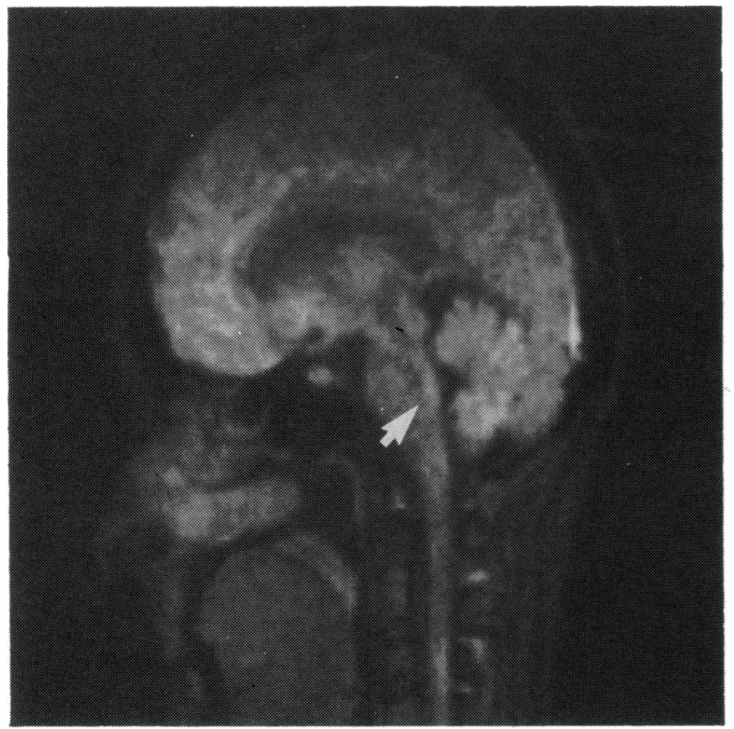

Fig. 4 A sagittal $T_{1}$ weighted image showing high signal from the area of pontine demyelination.
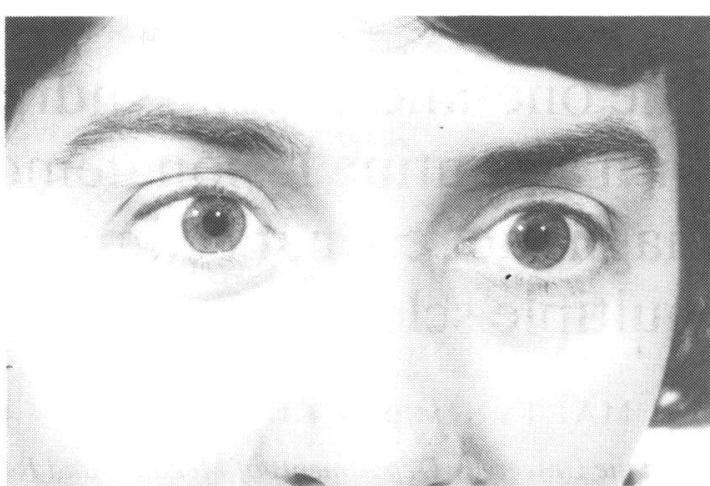

Fig. 3 Patient attempts to look left. There is no movement of either eye.

positive at 1:1000, VDRL and TPHA tests for syphilis negative on both blood and CSF, serum $B_{12}$ and folate normal. CSF protein was $282 \mathrm{mg} / \mathrm{l}$ and oligoclonal bands were not detected. The CSF cell count was $1 / \mathrm{mm}^{3}$. The CT brain scan was normal, and there were no abnormalities on chest $x$-ray. Visually evoked responses to a reversing checker board stimulus demonstrated a delay of the P100 wave in the right eye of $18 \mathrm{~ms}$ relative to the left.

Nuclear magnetic resonance imaging was performed on a low field $(0.08 \mathrm{~T})$ resistive MR system ${ }^{56}$ with a TR (repetition rate) of 1 second and a TI (time from inversion) of $200 \mathrm{~ms}$. Lesions such as those found in demyelinating conditions produce an elevation in magnetic relaxation times and were clearly shown by NMR. ${ }^{5}$ Figs. 4 and 5 are $T_{1}$ weighted images which show a left-sided abnormality in the lower

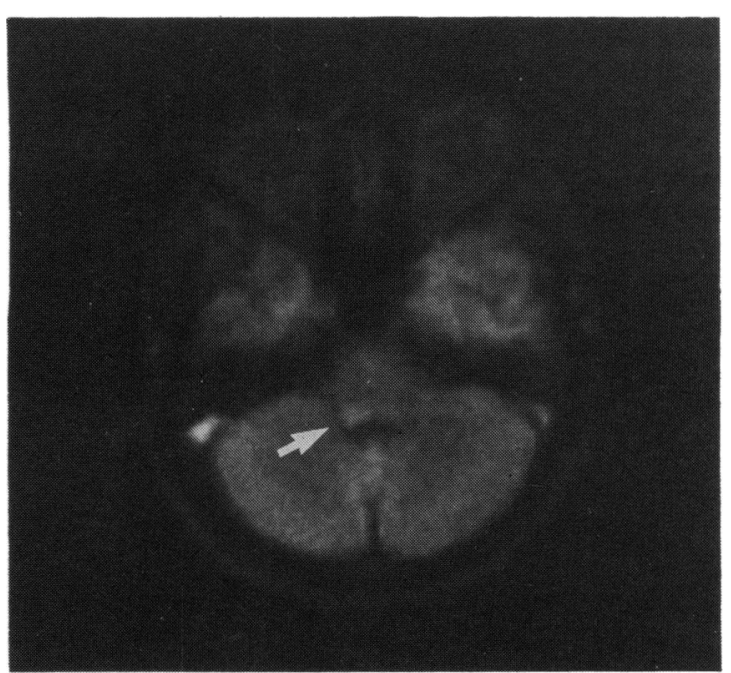

Fig. 5 A transverse $T_{1}$ weighted image showing that the lesion is confined to the left side of the dorsal pons. 
pontine tegmentum. This area of prolonged $T_{1}$ is typical of an area of demyelination.

The patient was treated with dexamethasone 8 $\mathrm{mg} /$ day for five days. Her facial palsy, loss of taste, and internuclear ophthalmoplegia recovered gradually over the next month. After six months her only remaining abnormality was vertical nystagmus on upgaze. She was re-examined by NMR, which, despite the clinical improvement, showed little change.

\section{Discussion}

This woman has sustained four episodes of neurological disturbance in the past nine years, recovering completely or nearly completely from each. There has never been any clinical or laboratory evidence of an arteritic condition or of sarcoidosis. Although there are no typical abnormalities in the CSF, the diagnosis is almost certainly multiple sclerosis.

The one-and-a-half syndrome is thought to be caused by a lesion in the lower pontine tegmentum affecting the sixth nerve nucleus and the lateral gaze centre in the adjacent paramedian reticular formation as well as the ipsilateral median longitudinal fasciculus. In this case the facial weakness and disturbance of taste indicate that the seventh nerve nucleus and the rostral part of the nucleus of the tractus solitarius, or alternatively the infranuclear fibres of the facial nerve and nervus intermedius as they pass through the pons, were also affected. A small lesion affecting exactly this area is clearly demonstrated by nuclear magnetic resonance imag- ing. This case shows the value of magnetic resonance imaging techniques in the investigation of brain stem lesions. Not only has it been possible to localise the lesion producing this unusual combination of disorders of eye movements within the pons but information about the pathology of the lesion has been provided. NMR allowed a definite diagnosis to be reached during the acute phase of the illness without recourse to invasive investigations.

We are grateful to Dr M A Smith, Miss R A B Douglas, and Mrs A Blane, of the NMR Imaging Unit, Royal Infirmary, Edinburgh, for their help with this case.

\section{References}

1 Freeman W, Ammerman HH, Stanley H. Syndrome of the pontile tegmentum: Foville's syndrome: report of three cases. Arch Neurol Psychiatry 1943; 50: 462-71.

2 Fisher CM. Some neuro-ophthalmological observations. J Neurol Neurosurg Psychiatry 1967; 30: 383-92.

3 Pierrot-Deseilligny C, Chain F, Serdaru M, Gray F, Lhermitte F. The one-and-a-half syndrome. Electro-oculographic analysis of five cases with deductions about the physiological mechanisms of lateral gaze. Brain 1981; 104: 665-99.

4 Wall M, Wray SH. The one-and-a-half syndrome-a unilateral disorder of the pontine tegmentum: a study of 20 cases and review of the literature. Neurology 1983; 33: 971-80.

5 Smith MA, Best JJK, Douglas RHB, Kean DM. The installation of a commercial resistive NMR imager. Br J Radiol 1984; 57: 1145-58.

6 Kean DM, Smith MA, Douglas RHB, Best JJK. Description of a low field resistive MRI system and its application in imaging midline central nervous system pathology. Clin Radiol in press.

Accepted for publication 18 May 1987. 